\title{
Autoignition and flame propagation in non-premixed MILD combustion
}

\author{
N. A. K. Doan ${ }^{\mathrm{a}, *}$, N. Swaminathan ${ }^{\mathrm{a}}$ \\ Submitted: 2 June 2018, Revised: December 21, 2018 \\ ${ }^{a}$ Department of Engineering, University of Cambridge, Trumpington Street, Cambridge, \\ CB2 1PZ, United Kingdom
}

\begin{abstract}
Direct Numerical Simulation (DNS) data of Moderate or Intense Low-oxygen Dilution (MILD) combustion are analysed to gather insights on autoignition and flame propagation in MILD combustion. Unlike in conventional combustion, the chemical reactions occur over a large portion of the computational domain. The presence of ignition and flame propagation and their coexistence are studied through spatial and statistical analyses of the convective, diffusive and chemical effects in the species transport equations. Autoignition is observed in regions with lean mixtures because of their low ignition delay times and these events propagate into richer mixtures either as a flame or ignition. This is found to be highly dependent on the mixture fraction length scale, $\ell_{Z}$, and autoignition is favoured when $\ell_{Z}$ is small.
\end{abstract}

Keywords: DNS; MILD combustion; Ignition; Flameless combustion; Flux analysis

\footnotetext{
${ }^{*}$ Currently at Technical University of Munich

Email address: doan@tfd.mw.tum.de (N. A. K. Doan)
} 


\section{Introduction}

Moderate or Intense Low-oxygen Dilution (MILD) combustion has been identified as a potential concept to simultaneously improve the energy efficiency of combustion devices and reduce their pollutant emissions $[1,2]$. This concept relies on preheating and dilution of reactant mixture. The reactants are typically preheated to a temperature, $T_{r}$, higher than their autoignition temperature, $T_{i g n}$, and the increase in temperature during combustion, $\Delta T=\left(T_{p}-T_{r}\right)$, is smaller than $T_{i g n}[2]$. These conditions are achieved typically by Exhaust Gas Recirculation (EGR) to preheat the reactants and to decrease the oxygen content.

Early experiments used direct photographs or laser thermometry to identify features of MILD combustion [3-5] showing a spatially uniform combustion without a clearly visible flame. However, more advanced techniques like OH-PLIF have shown the existence of thin zones with clear $\mathrm{OH}$ gradient [6-8] suggesting the existence of flames. Also, autoignition is likely to play a major role given that $T_{r}>T_{i g n}[1,2,9]$. This was studied in a Jet in Hot Coflow (JHC) configuration using $\mathrm{CH}_{2} \mathrm{O}-\mathrm{PLIF}[7,10]$ and it was observed that the heated coflow played a key role in sustaining the combustion by initiating ignition after localized extinction caused by entrainment of the surrounding cold air. Despite this interplay between extinction and ignition, the abundance of hot exhaust gas ensured the sustained combustion. Oldenhof et al. [11] analysed the evolution of these ignition kernels using UVluminescence and observed that ignition kernels were produced constantly, and grew in size as they were convected downstream. Similar observations were reported in jet in hot crossflow [12]. The importance of autoignition 
appearing at the most reactive mixture fraction, $Z_{\mathrm{MR}}$, was also noted in [13] at the flame base. These kernels then propagated towards stoichiometric and richer mixture. These observations suggest a strong role of autoignition and flame propagation in MILD combustion $[2,14]$ and this needs to be investigated further to improve our understanding of MILD combustion since this will have a large impact for the modelling of this combustion. The modelling approach should be able to capture both autoignition and flame-propagation phenomena.

Direct Numerical Simulations (DNSs) are conducted recently to provide some insights into these specific aspects of MILD combustion. Past DNS of a mixing layer of methane and diluted-hot air highlighted the existence of autoignition along the iso-surface of most reactive mixture fraction rather than propagating flames $[15,16]$. These ignition events were found to depend on the local temperature and scalar dissipation rate. Other DNS of premixed MILD combustion with internal EGR [17-20] showed frequent interaction of reaction zones leading to their apparent broadening yielding the so-called flameless appearance for MILD combustion. Furthermore, the autoignition and flames were present together. The local behaviour of scalar gradient played a major role in determining whether ignition or flame propagation was favoured. The mixture fraction variations in EGR-type configuration, similar to HODO of [21], showed ignition along with premixed and nonpremixed flames in MILD combustion [22].

The objective of the present work is thus to shed further light on the autoignition and flame aspects in the MILD combustion with mixture fraction variation. This is achieved by studying the balance among various fluxes in 
the species transport equation using the DNS data of [22].

The paper is organised as follows. The DNS data and its methodology is explained briefly in section 2. The study of ignition, flame-propagation and their relative contribution are discussed in section 3. Conclusions are summarised in the final section.

\section{MILD combustion DNS data used}

DNS data generated in [22] for non-premixed MILD combustion with internal EGR is used for this work. Its detailed description is in [22] and a brief summary is provided below. The DNS considered non-premixed MILD combustion of methane in air diluted with exhaust gases at atmospheric pressure inside a cubic domain. A schematic representation of the procedures used are shown in Fig. 1. The preprocessing steps 1 to 5 dealt with generating the initial flow, mixture fraction, $Z$, reaction progress variable, $c$, and scalar mass fraction, $Y_{i}$, fields. A decaying homogeneous isotropic turbulence was simulated in step 1 to get the required turbulence field. Laminar premixed flames under MILD conditions for various values of $Z$ were computed and the scalar mass fractions were then mapped onto $Z$ and $c$ in step 2. The

initial turbulent mixture fraction, $\widehat{Z}$, and reaction progress variable, $\widehat{c}$, fields having prescribed means, $\langle Z\rangle$ and $\langle c\rangle$, and length scales, $\ell_{Z}$ and $\ell_{c}$ were constructed in step 3. The mixture fraction was defined using Bilger's definition [23] and $c$ was defined using the fuel mass fraction. The scalar mass fractions obtained as $Y_{i}(Z, c)$ in step 2 are then mapped onto the turbulent $Z$ and $c$ fields generated in step 3. This mapping was done in step 4 . The flow and scalar fields generated as above were then allowed to interact in 
step 5 for about one large eddy turn over time (smaller than the lowest ignition delay time) to ensure that the scalar and flow fields had interacted so that the velocity-scalar correlations discussed in [24-26] had evolved sufficiently before combustion has begun. The scalar field obtained at the end of step 5 included unburnt $(c=0)$, burnt $(c=1)$ and also partially burnt (intermediate values of $c$ ) mixtures with the equivalence ratio ranging from 0 to 10 inside the computational domain. The equivalence ratio is defined as $\widehat{\phi}=\widehat{Z}\left(1-\widehat{Z}_{s t}\right) /\left(\widehat{Z}_{s t}(1-\widehat{Z})\right)$, where $Z_{s t}$ is the stoichiometric mixture fraction. These fields were then used as the initial and inflowing conditions for the combustion DNS in the second stage as shown in Fig. 1. The interested reader is referred to [22] for elaborate detail on these procedures.

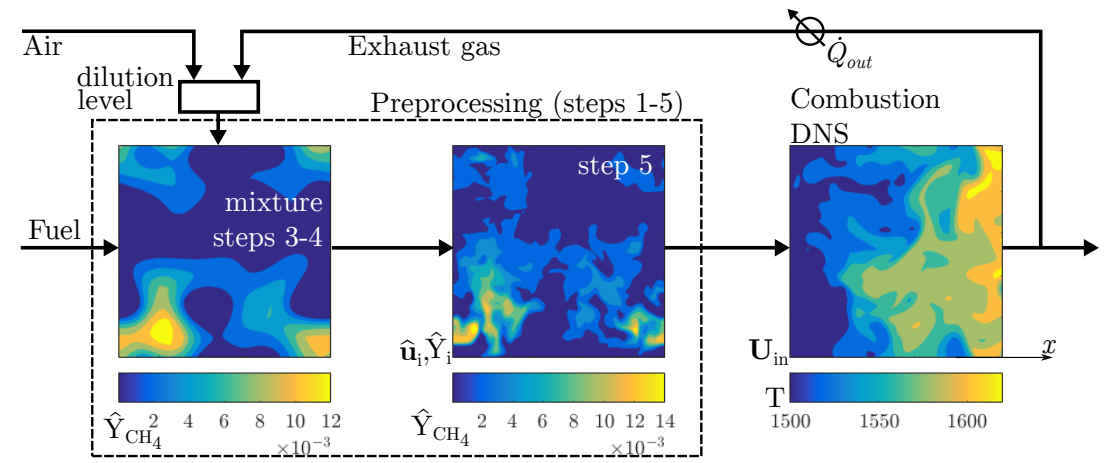

Fig. 1. Schematics of DNS steps followed for non-premixed MILD combustion with internal recirculation of exhaust gases (from [22]).

Table 1. Oxidiser composition for the initial laminar MILD mixture.

\begin{tabular}{lllll}
\hline Case & $X_{\mathrm{O}_{2}, o x}$ & $X_{\mathrm{H}_{2} \mathrm{O}, o x}$ & $X_{\mathrm{CO}_{2}, o x}$ & $X_{\mathrm{N}_{2}, o x}$ \\
\hline AZ1 \& AZ2 & 0.035 & 0.134 & 0.067 & 0.764 \\
BZ1 & 0.020 & 0.146 & 0.073 & 0.761 \\
\hline
\end{tabular}


Three cases detailed in Tables 1 and 2 were simulated [22]. The cases AZ1 and AZ2 used the same oxidiser with 3.5\% (by volume) of $\mathrm{O}_{2}$ while the case BZ1 had a more diluted oxidiser $\left(2 \%\right.$ of $\left.\mathrm{O}_{2}\right)$ as listed in Table 1. The cases AZ1 and AZ2 differed by the lengthscale ratio, $\ell_{Z} / \ell_{c}$, to study its influence on MILD combustion. The case $\ell_{Z} / \ell_{c}<1$ was not considered because the mixing length scale (of the mixture fraction field) is generally larger than the chemical length scales such as the flame thickness or ignition kernel size at $T_{r}$ as large as $1500 \mathrm{~K}$. The turbulence field for all cases was similar with an integral length scale of $\Lambda_{0} \approx 1.42 \mathrm{~mm}$ and root-mean square value of $u^{\prime} \approx 16.66 \mathrm{~m} / \mathrm{s}$ for the velocity fluctuations. This yielded turbulence and Taylor microscale Reynolds numbers of $\operatorname{Re}_{t} \approx 96$ and $\operatorname{Re}_{\lambda} \approx 34.73$ respectively.

Table 2. DNS initial conditions.

\begin{tabular}{cccccccccc}
\hline Case & $\Lambda_{0} / \ell_{Z}$ & $\left\langle X_{\mathrm{O}_{2}}\right\rangle$ & $X_{\mathrm{O}_{2}}^{\max }$ & $\ell_{Z} / \ell_{c}$ & $\langle Z\rangle$ & $Z_{s t}$ & $\sigma_{Z}$ & $\langle c\rangle$ & $\sigma_{c}$ \\
\hline AZ1 & 0.60 & 0.0270 & 0.035 & 1.30 & 0.008 & 0.010 & 0.0084 & 0.56 & 0.26 \\
AZ2 & 0.79 & 0.0285 & 0.035 & 1.01 & 0.008 & 0.010 & 0.0105 & 0.56 & 0.28 \\
BZ1 & 0.60 & 0.0160 & 0.020 & 1.30 & 0.0046 & 0.0058 & 0.0057 & 0.56 & 0.26 \\
\hline
\end{tabular}

The numerical domain was a cube of size $L_{x} \times L_{y} \times L_{z}=10 \times 10 \times 10 \mathrm{~mm}^{3}$ with inflow and non-reflecting outflow boundary conditions in the $x$-direction and periodic conditions in the transverse, $y$ and $z$, directions. The domain was discretised using $512 \times 512 \times 512$ uniformly distributed grid points which ensured that all chemical and turbulence lengthscales were resolved [22]. The methane-air combustion chemistry is described using the MS-58 mechanism detailed in [22]. This mechanism is a modified version of the Smooke and 
Giovangigli mechanism [27] with $\mathrm{OH}^{*}$ chemistry from [28]. The elementary reactions involving precursors for $\mathrm{OH}^{*}$-chemistry were taken from the KEE-58 mechanism [23]. The MS-58 mechanism listed in [22] involved 19 species and 58 reactions, and balanced the accuracy and computational cost by giving a good agreement for laminar flame speed and ignition delay with measurements. Detailed validations of this mechanism are available in [22].

The numerical code SENGA2 solving fully compressible conservation equations for mass, momentum, internal energy and species mass fractions, $Y_{i}$, was used. A tenth order central difference scheme was used for spatial discretisation at each mesh point. Close to the boundaries, this is progressively reduced to a fourth-order scheme for non-periodic boundary points. Inlet and outlet boundary conditions are specified using the Navier-Stokes Characteristic Boundary Conditions (NSCBC) [29]. The time integration is performed using a low-storage third order Runge-Kutta scheme. The transport and thermo-chemical properties were temperature dependent with non-unity constant Lewis numbers. Each case was run for about 1.5 flow-through time, $\tau_{f}=L_{x} / U_{\text {in }}$ where $U_{\text {in }}=20 \mathrm{~m} / \mathrm{s}$ was the inflowing velocity, using a timestep of $\delta t=1$ ns. Statistics were taken after the first flow-through time to ensure that the initial transients had left the domain and the analysis reported below were conducted using 50 snapshots. These simulations were run on ARCHER, a Cray XC30 system, and each simulation took approximately 550 wall-clock hours using 4096 cores. 


\section{Autoignition and flame propagation phenomena}

MILD combustion involves a mixture of fuel and hot diluted oxidiser in unburnt, burnt and partially burnt states. This range of mixture states is produced by incomplete mixing and combustion of the fuel and oxidiser. This produces a large fluctuation in the local mixture fraction, reaction progress variable and mass fractions of radicals and intermediates, which will have a strong impact on the development of autoignition or flames. Furthermore, the local turbulence will also have a large influence on this. These are studied using a flux-balance analysis which allows to identify regions dominated by reactions (autoignition) or convective-diffusive-reactive balance (flames).

\subsection{Balance of convection, diffusion and reaction}

The transport equation for the mass fraction of species $\alpha$ is given by

$$
\frac{\partial \rho Y_{\alpha}}{\partial t}+\underbrace{\frac{\partial \rho u_{j} Y_{\alpha}}{\partial x_{j}}}_{\mathcal{C}: \text { conv. }}=\underbrace{\frac{\partial}{\partial x_{j}}\left(\rho D_{\alpha} \frac{\partial Y_{\alpha}}{\partial x_{j}}\right)}_{\mathcal{D}: \text { diff. }}+\underbrace{\dot{\omega}_{\alpha}}_{\mathcal{R}: \text { react. }}
$$

where $u_{j}$ is the velocity in direction $j, D_{\alpha}$ is the molecular diffusivity of the species $\alpha$ and $\dot{\omega}_{\alpha}$ is its reaction rate. Turbulent combustion is thus a balance among convection, diffusion and chemical reactions. Following [19], this balance can be studied by analysing $\mathcal{B}$ defined as:

$$
\mathcal{B} \equiv|\mathcal{C}-\mathcal{D}|-|\mathcal{R}|
$$

The species transport equation can then be written as $\partial \rho Y_{\alpha} / \partial t+\mathcal{B}=0$. Equation (2) suggests that regions with $\mathcal{B}<0$ have dominant reaction source. These regions are taken to be similar to Perfectly Stirred Reactors (PSRs) and thus they are ignition dominated regions. There is a balance among 
convection, diffusion and reaction in a steady laminar flame and thus $\mathcal{B}=0$ in regions with flames. However, the large velocity fluctuations can lead to flame displacements, which translates into large $\mathcal{C}$. Hence, in the presence of turbulence, regions with unsteady flames are characterised with $\mathcal{B}>0$. Furthermore, non-reacting regions will have $\mathcal{B}>0$ because $\mathcal{R}=0$. Thus, the $\mathcal{B}$ field needs to be conditioned using the heat release rate for an unambiguous identification of autoignition or flame. The mass fraction of $\mathrm{H}_{2} \mathrm{O}$ is used for this flux analysis and investigations using $Y_{\mathrm{CH}_{4}}$ or other species yielded results similar to those discussed below.

Figure 2 shows the variations of $\mathcal{B}$ in regions with $\dot{Q}^{+} \geq 1$ for the three cases listed in Table 2, where $\dot{Q}^{+}=\dot{Q} \delta_{t h} /\left(\rho_{r} s_{L} C_{P}\left(T_{p}-T_{r}\right)\right)$ is the normalised heat release rate per unit volume. The normalisation is done using the thermo-chemical quantities for the local mixture fraction; $\rho_{r}$ is the unburnt mixture density, $\delta_{t h}$ is the laminar flame thermal thickness, $s_{L}$ is the laminar flame speed, $C_{p}$ is the constant pressure specific heat capacity, and $T_{p}$ and $T_{r}$ are respectively the product and reactant temperatures. It is observed that reaction zones are spread over a substantial portion of the domain in all cases and it increases with dilution level as seen in Fig. 2. However, the cases AZ1 and AZ2 suggest the existence of thin reaction zones. Figure 2c shows large regions with $\mathcal{B}<0$ implying the dominance of autoignition contribution in the case BZ1.

\subsection{Lagrangian tracking of fluid parcels and their combustion modes}

The evolution of $\mathcal{B}$ is tracked using Lagrangian particles to study the mixed ignition-flame behaviour. Specifically, how a fluid parcel undergoes ignition or develops into a propagating flame solely based on the quantity $\mathcal{B}$. 

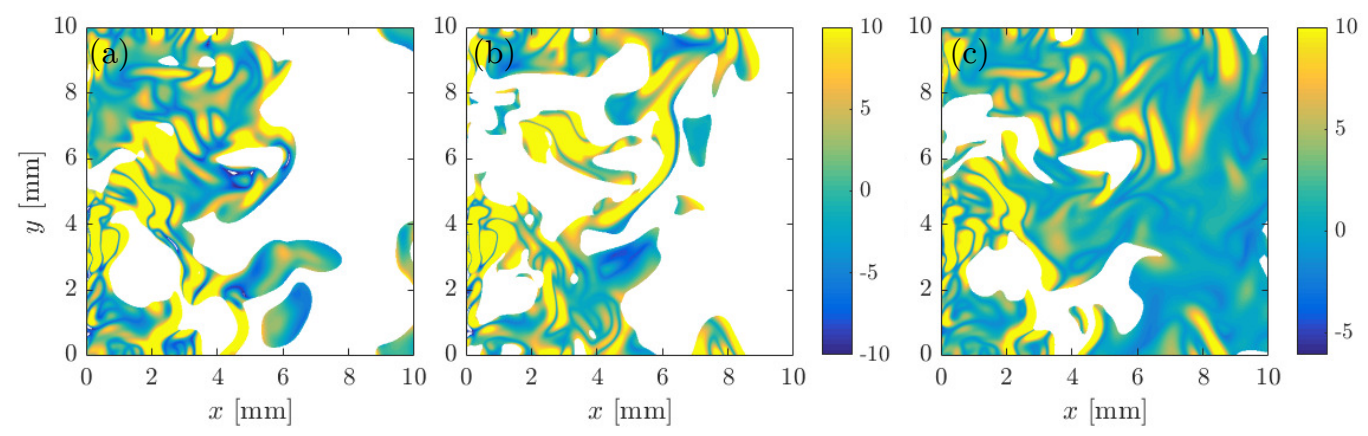

Fig. 2. Typical spatial variation of $\mathcal{B}$ in regions with normalised heat release rate, $\dot{Q}^{+}$, larger than 1.0 at $t=1.5 \tau_{f}$ for cases (a) AZ1, (b) AZ2 and (c) BZ1.

The analysis of local structures showed that using $\mathcal{B}$ is sufficiently accurate for this purpose and thus the local spatial structures at a given time are not shown in this paper. The Lagrangian particles are seeded in four different axial planes located at $x=0, L_{x} / 4, L_{x} / 3$ and $L_{x} / 2$ for $t=\tau_{f}$. Their spatial locations are tracked in time through $\underline{x}(t+\Delta t) \approx \underline{x}(t)+\underline{u}(t) \Delta t$ where $\Delta t=4.5 \mu \mathrm{s}$ is the time difference between the two saved DNS snapshots. This is performed for the entire sampling duration of $\tau_{f} \leq t \leq 1.5 \tau_{f}$. The particles are injected at every grid points in each of these four planes, which gives $512 \times 512$ particles per plane and about 1 million particles in total. All of these particles are tracked individually in time and the evolution of key quantities along their paths are carefully analysed here.

One could have used the DNS timestep of $\delta t=1 \mathrm{~ns}$ instead of $\Delta t$. However, a comparison of the particle locations obtained using $\Delta t=1 \mathrm{~ns}$ (by rerunning the DNS for a short period of $4.5 \mu \mathrm{s}$ ) with those obtained using $\Delta t=4.5 \mu$ s showed an average relative difference of about $0.02 \%$ for the particle location and various quantities analysed. Thus, $\Delta t=4.5 \mu \mathrm{s}$ was 
used for the analysis reported here. This choice avoids rerunning the entire DNS with particles and the very small difference for the location (0.02\%) observed is because the velocity field is not unduly influenced by the local heat release rate. However, the fluid mixture state at location $\mathbf{x}(t+\Delta t)$ for time $(t+\Delta t)$ was computed using $\delta t$ in the DNS and thus it has evolved under the influences of convection, diffusion and reaction.

Figure 3 shows few typical trajectories for all the cases and those highlighted using arrows are investigated further in Figs. 5 to 7 . The similarities of various trajectories shown for the three cases are because these DNSs are initialised using the same turbulence field. Also, the velocity field is not strongly influenced by the small heat release rate. However, the evolution of $\mathcal{B}$ along these trajectories are quite different for the three cases because of the difference in the local mixture conditions arising from turbulent mixing and dilution. The projections of their trajectories in the $x-y$ and $x-z$ planes are presented in Fig. 4 for the case AZ1 to show that these particles move in 3D space. The individual trajectories are quite different since the flow is turbulent and the lengths of these trajectories are indicative of the local velocities (longer trajectory implies higher velocity). There is a large variation of $\mathcal{B}$ along the trajectories and thus these fluid parcels undergo ignition and/or flame propagation phenomena. This $\mathcal{B}$ field is obtained separately using DNS quantities (see Eqs. 1 and 2) and is not influenced by the Lagrangian tracking procedure. Thus, the colour change represents the time variation of $\mathcal{B}$ on these trajectories and hence this should not be compared directly with the results shown in Fig. 2. Out of these trajectories, a few particles exhibiting representatives evolutions are annotated in Fig. 4 which are also shown by 
arrows in Fig. 3. These are selected for further analysis and the starting locations of these trajectories are listed in Table 3. These tracked fluid elements undergo both ignition and flame phenomena indicated by the change in the $\mathcal{B}$ value, which are obtained independently of the tracking procedure as noted earlier.

Table 3. Initial locations of the tracked particles $\left(x / L_{x}, y / L_{y}, z / L_{z}\right)$ highlighted in Fig. 3.

\begin{tabular}{cccc}
\hline & AZ1 & AZ2 & BZ1 \\
\hline a & $(0.250,0.225,0.008)$ & $(0.250,0.008,0.002)$ & $(0.250,0.241,0.969)$ \\
b & $(0.500,0.006,0.583)$ & $(0.333,0.288,0.816)$ & $(0.000,0.231,0.315)$ \\
c & $(0.333,0.245,0.796)$ & & \\
\hline
\end{tabular}

Figure 5 shows the spatial evolution of $\dot{Q}, Y_{\mathrm{CH}_{4}},|\nabla Z|,\left|\nabla c_{T}\right|$ and $\Delta T=$ $T-T_{r}$ along with the variations of $\mathcal{B}$ and $\phi$ for the three trajectories of the case AZ1 marked in Figs. 3 and 4. These quantities, except for $\mathcal{B}$ and $\phi$, are normalised using their respective maximum along the chosen trajectory and are denoted with a superscript * These are plotted as a function of the streamwise direction only but the particles are moving in the transverse, $y$ and $z$, directions as shown in Figs. 3 and 4. Furthermore, as their starting axial locations are different the range of $x$ on each frame of Fig. 5 is different. The quantities shown are for the period starting from $t=\tau_{f}$ to $1.5 \tau_{f}$. The starting location of the tracking corresponds to the left side of the $x$-axis while the end is on the right. Furthermore, $\mathcal{B}$ is only computed in regions where $\dot{Q}^{+}>1$ (and plotted as solid black line) to emphasis reacting regions. Otherwise, $\mathcal{B}$ is plotted using a dotted line having a value of 0 .

These specific particles were chosen because they illustrate the statistical 

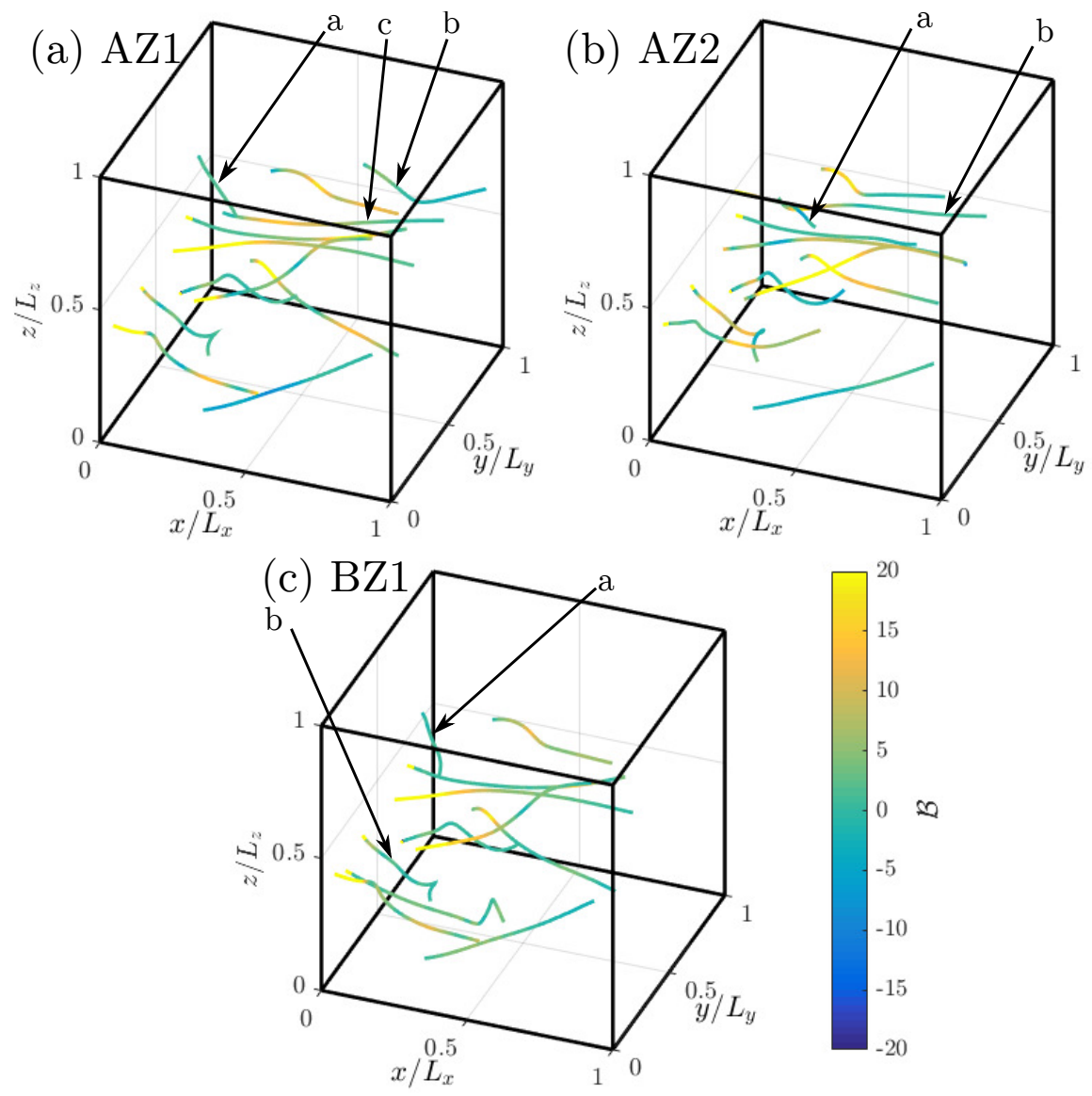

Fig. 3. Typical trajectories of Lagrangian particles tracked for $\tau_{f} \leq t \leq 1.5 \tau_{f}$ for all cases. Lines are coloured using $\mathcal{B}$ values along the trajectory. Arrows indicate trajectories analysed further later in Figs. 5 to 7.

evolution of $\mathcal{B}$ to be discussed later in section 3.3. Figure 5a shows the evolution of a fluid element undergoing ignition and then developing into a propagating flame structure. Indeed, $\mathcal{B}$ starts as negative indicating that reactions are dominant (see Eq. 2) and thus there is ignition. Then, there is an increase of $\mathcal{B}$ towards zero which is representative of a steady flame. The ignition occurs in a lean region $(\phi<1)$ close to the inlet, $x / L_{x} \approx 0.25$, 

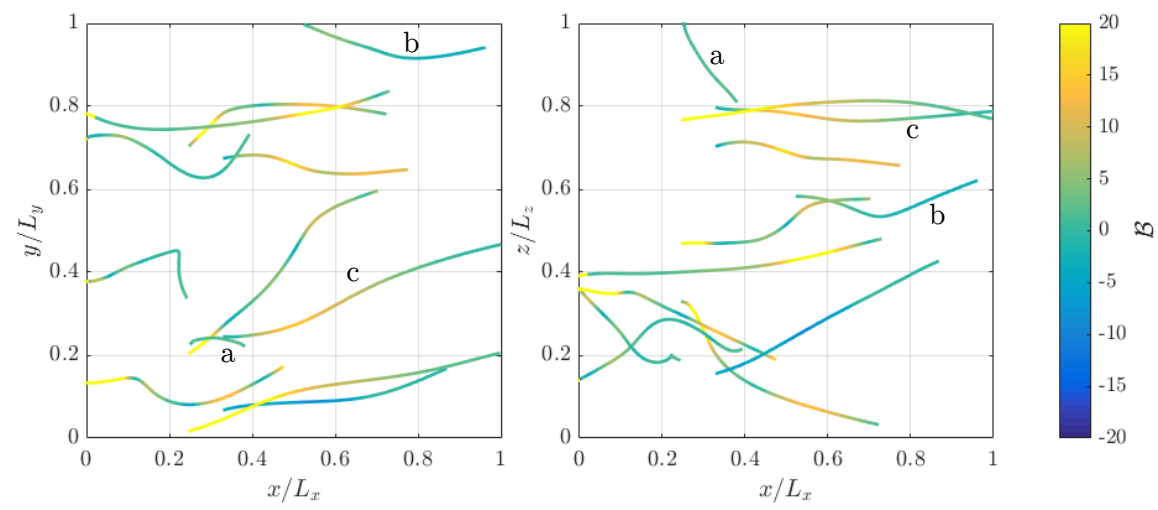

Fig. 4. Projection on the $x-y$ and $x-z$ planes of the trajectories in Fig. 3a. Lines are coloured using $\mathcal{B}$ values along the trajectory. Annotated trajectories are analysed further in Fig. 5.

which is due to the high reactivity of lean mixtures and their relatively small ignition delay time [16] (see Fig. 11 to be discussed later). This ignition kernel propagates through a slightly richer mixture in the downstream, as indicated by the increase in $\phi$ and it develops into a flame as indicated by $\mathcal{B} \rightarrow 0$. The quantity $\left|\nabla c_{T}\right|$ is high at the beginning because of relatively larger $c_{T}$ inside the ignition spot compared to its surrounding with unburned mixture $\left(c_{T} \approx 0\right)$. Also, the mixture fraction distribution around this kernel yields large $|\nabla Z|$. Ignition occuring at this location with high $|\nabla Z|$ and scalar dissipation rate, $N_{Z}=D_{Z}|\nabla Z|^{2}$, is in contrast to the conventional combustion with ignition occurring in regions of low $N_{Z}$. This contrasting behaviour for MILD combustion is because of the presence of radicals as noted in [30]. As the parcel is convected downstream, its surrounding mixture having relatively uniform $c_{T}$ also starts to react and thus $\left|\nabla c_{T}\right|$ decreases. The evolution of $|\nabla Z|$ gives an indication of the small scale mixing. It is seen that there are two instances of large $|\nabla Z|$ and the second local maximum of 

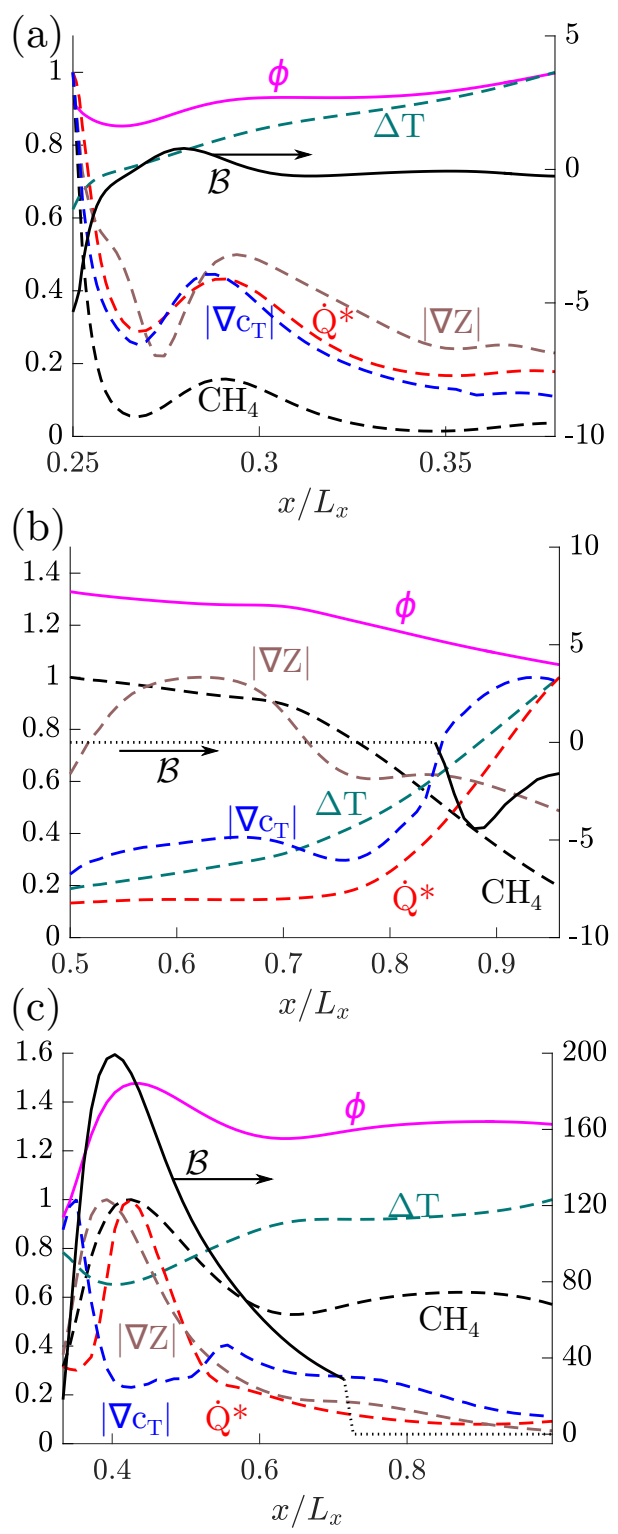

Fig. 5. Evolution of $\dot{Q}^{*}, \phi, Y_{\mathrm{CH}_{4}}^{*}, Y_{\mathrm{O}_{2}}^{*},|\nabla Z|^{*},\left|\nabla c_{T}\right|^{*}, \Delta T^{*}$, (left axis) and $\mathcal{B}$ (right axis) along the three trajectories of case AZ1, see Figs. 3 and 4.

$|\nabla Z|$ results from the fluid parcel mixing with a richer mixture (increasing $\phi)$. This provides the fuel required to sustain the chemical reactions, which 
is seen from the variations of $Y_{\mathrm{CH}_{4}}$ and $\dot{Q}$ shown in Fig. 5a. There is a progressive increase in temperature throughout this process. The variations shown in Fig. 5a depicting the history of the chosen fluid parcel illustrate the ignition of a lean mixture in an upstream location developing into a propagating flame downstream.

Figure 5b illustrates another behaviour namely, the ignition of a rich mixture in the downstream region. The rich mixture is weakly reacting in the early stage as indicated by the low heat release rate despite the presence of both $\mathrm{CH}_{4}$ and $\mathrm{O}_{2}$ (not shown here for clarity of Fig. 5). This is due to the low reactivity and large ignition delay time of this rich mixture (to be discussed later in Fig. 11). However, this rich parcel undergoes mixing in the early parts of the domain which is indicated by the relatively large and nearly constant $|\nabla Z|$, slow drop in $Y_{\mathrm{CH}_{4}}$, slow increase in $\Delta T$ but no change in $\dot{Q}^{*}$. This decreases the equivalence ratio and gradually heats up the parcel $(\Delta T$ increases slowly), leading to its ignition. This is seen by the large increase of $\dot{Q}^{*}$ and the decrease of $Y_{\mathrm{CH}_{4}}$ further downstream. Thus, $\mathcal{B}$ starts to become negative as seen in Fig. 5b. The large value of $\left|\nabla c_{T}\right|$ in the downstream is because this parcel is surrounded by burned mixture while it begins to react. The $\left|\nabla c_{T}\right|$ increases sharply once the fluid parcel ignites $\left(x / L_{x}=0.83\right)$.

Figure 5c presents the evolution of a rich flame-like structure. This parcel, from a flame around stoichiometry $\left(\mathcal{B} \approx 0, \phi \approx 1\right.$, with decreasing $\left.\dot{Q}^{*}\right)$, propagates towards an unburnt rich mixture (increasing value of $Y_{\mathrm{CH}_{4}}$ ). The high $\left|\nabla c_{T}\right|$ value indicates that the mixture surrounding the tracked parcel have a different $c_{T}$, smaller here verified separately, and $|\nabla Z|$ is large too indicating the flux of mixture fraction and thus mixing. This is also confirmed 
by the small decrease in $\Delta T$ which indicates that the original parcel has mixed with cooler mixture. This mixing is also emphasized by the large increase in $\mathrm{CH}_{4}$. As a result of this large-scale mixing which is driven by the convective effects, $\mathcal{B}$ increases to large positive values and the flame continues to propagate towards richer mixture.

Comparing these three evolutions, an important aspect to highlight is in their streamwise locations. Indeed, the ignition of the lean parcel, and its subsequent evolution towards a propagating flame shown in Fig. 5a occurs in the early part of the domain $\left(x / L_{x} \approx 0.25\right)$ while the ignition of the rich parcel occurs farther downstream $\left(x / L_{x} \approx 0.9\right)$. This difference is explained by their different ignition delay times (to be shown later in Fig. 11). The temperature increase for each parcel is extremely progressive which is a key characteristics of MILD combustion where there is no sharp or sudden increase of temperature.

These three behaviours illustrate several key characteristics for the case AZ1 which will be re-emphasized in the next subsection. Mainly, lean mixtures show autoignition behaviour which evolves towards a flame while rich mixtures are either autoigniting in the downstream or react through an initially lean flame propagating towards them. The existence of flames observed here is contrasted to the autoignition along the iso-surface of most reactive mixture fraction reported in the DNS of an autoigniting mixing layer [16]. Finally, a stronger flame in the initial stages can become relatively weaker because of large fuel flux coming from mixing with surrounding richer mixtures.

For case AZ2, the most probable behaviour observed corresponds to 
ignition-like events which is in contrast to the case AZ1 discussed above. This is illustrated in Fig. 6 showing the evolution of various quantities for the parcels tracked (see Fig. 3b). Figure 6a shows the ignition of a very lean mixture $(\phi \approx 0.17)$. Unlike the lean parcel in the case AZ1 shown in Fig. 5a, this mixture in the case AZ2 has nearly no $\mathrm{CH}_{4}$ and thus it is convected downstream mixing with richer mixture which makes it ignitable. The sharp rise in $\left|\nabla c_{T}\right|$ is because of this ignition. A particular behaviour is also observed with a double peak of $\mathcal{B}$. Indeed, after ignition takes place (the first negative peak of $\mathcal{B}$ ), the $\mathcal{B}$ value increases towards $\mathcal{B}=0$ but instead of stabilising there, $\mathcal{B}$ becomes negative again indicating a second ignition-like event. This is emphasised by the existence of a double peak for the heat release rate. This is because the igniting parcels encounters a large mixture fraction stratification (indicated by the large $|\nabla Z|$ and progressive increase in $\phi)$. This kind of sequential ignition is due to the stratification in the mixture fraction and is also found in PCCI combustion with stratified mixture [31]. There is no such sequential ignition for the case AZ1 shown in Fig. 5a because the mixture stratification is relatively small (making it closer to HCCI conditions) where the development of ignition towards deflagration is more likely [31]. Figure $6 \mathrm{~b}$ shows the ignition behaviour of a rich mixture which is similar to that observed in Fig. 5b. This difference between case AZ2 and case AZ1 will be further studied in section 3.3 where it will be shown that for the case AZ2, ignition is the most probable combustion mode.

Figure 7 shows the evolution of two typical parcels for the case BZ1. In both cases, $\mathcal{B}$ starts negative indicating an ignition-like behaviour before increasing towards positive or zero values as the parcels move downstream. 


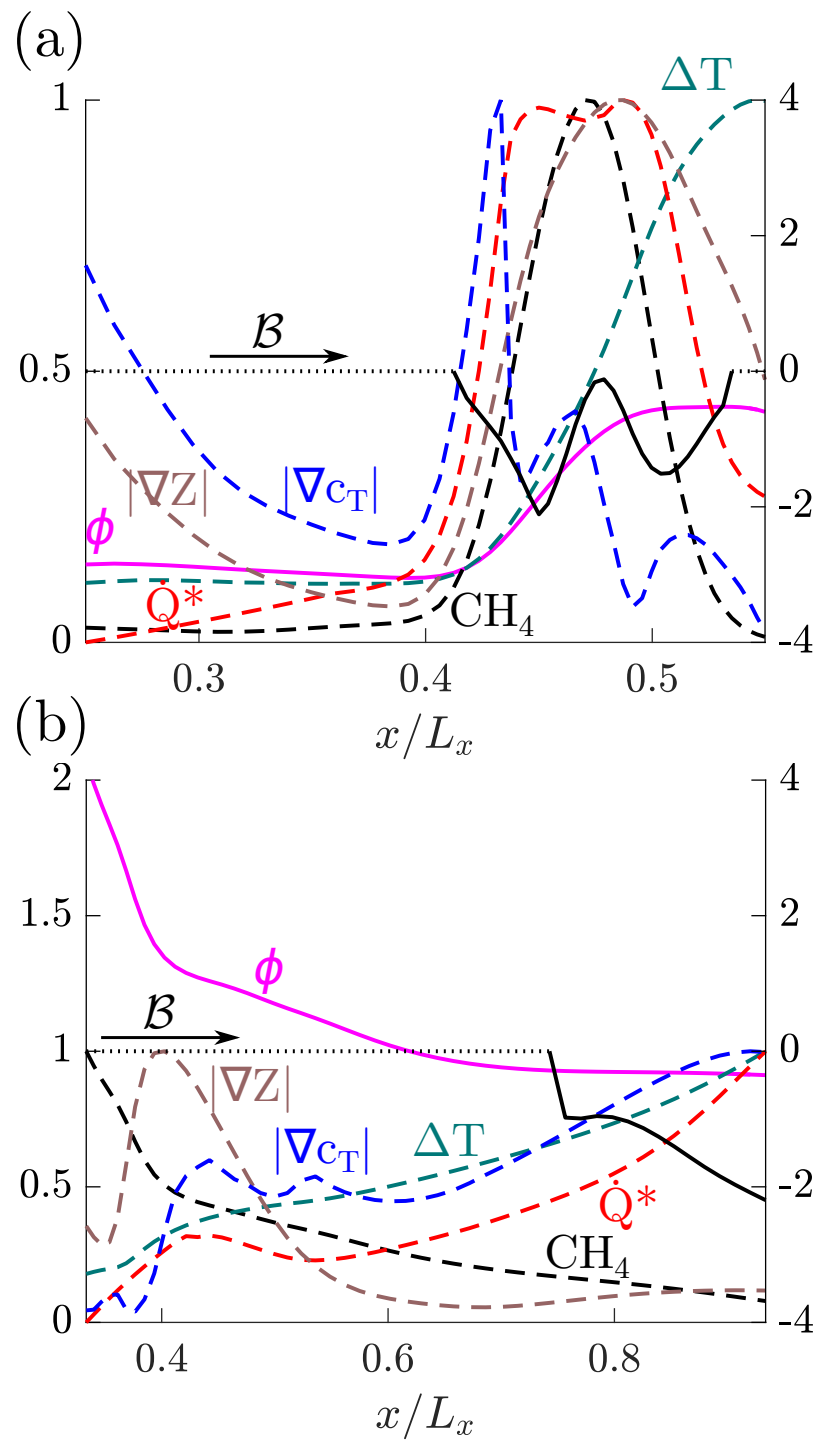

Fig. 6. Evolution of $\dot{Q}^{*}, \phi, Y_{\mathrm{CH}_{4}}^{*}, Y_{\mathrm{O}_{2}}^{*},|\nabla Z|^{*},\left|\nabla c_{T}\right|^{*}, \Delta T^{*}$ (left axis) and $\mathcal{B}$ (right axis) along the two trajectories of case AZ2 marked in Fig. 7.

However, Fig. 7b shows a more flame-like behaviour with a nearly constant heat release rate while Fig. 7a shows a decrease in heat release rate after ignition because reactions are slowing down. This particular behaviour is 
explained by the difference in mixture fraction between the two parcels. Indeed, a lean reacting parcel propagates towards a richer mixture having a relatively lower reactivity in Fig. 7a. Conversely, a lean parcel propagates in a mixture with nearly constant mixture fraction in Fig. 7b. The large and sharp variations around $x / L_{x}=0.4$ in Fig. $7 \mathrm{~b}$ are due to the projection of the trajectory on the $x$ axis. This parcel is inside a recirculating flow and has a nearly zero streamwise velocity (as it is moving in the $y$ and $z$ direction as observed in Fig. 3c).

Table 4. Summary of the combustion modes observed in Figs. 5 to 7.

\begin{tabular}{cccc}
\hline Trajectory & AZ1, Fig. 5 & AZ2, Fig. 6 & BZ1, Fig. 7 \\
\hline & Ignition of lean & Sequential ignition & \\
a & mixture evolving & of stratified & Lean ignition \\
& into flame & lean mixtures & \\
b & Ignition of & Ignition of & Lean ignition \\
& rich mixture & rich mixture & evolving to flame \\
& & & \\
c & Unsteady & & \\
\hline
\end{tabular}

The varieties of combustion behaviour observed in Figs. 5 to 7 are summarised in Table 4 for convenience. Now, the question arising is which one is the most probable in MILD combustion and how these various modes are balanced? 

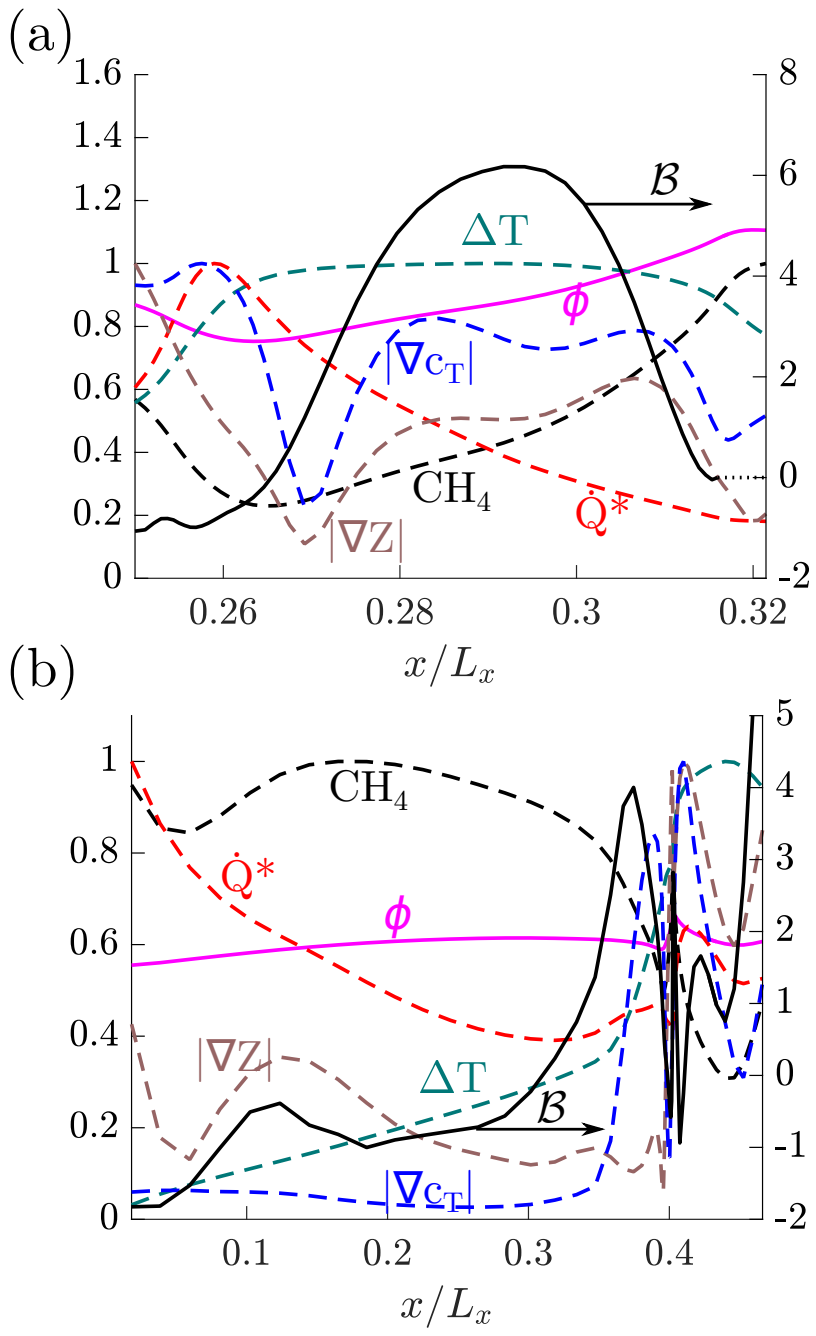

Fig. 7. Evolution of $\dot{Q}^{*}, \phi, Y_{\mathrm{CH}_{4}}^{*}, Y_{\mathrm{O}_{2}}^{*},|\nabla Z|^{*},\left|\nabla c_{T}\right|^{*}, \Delta T^{*}$, (left axis) and $\mathcal{B}$ (right axis) along the two trajectories of case BZ1 marked in Fig. 7.

\subsection{Statistical behaviour of ignition and flame modes}

The previous discussion highlights the existence of various combustion modes and most reaction zones show both autoignition $(\mathcal{B}<0)$ and flame $(\mathcal{B} \geq 0)$ behaviours. The relative importance of ignition and flame, and their correlations with the mixture fraction, $Z$, can be investigated in a statistical 
sense by studying the joint probability density function (PDF) of $\mathcal{B}$ and $Z$ at various axial locations. The joint PDF is constructed using the data collected over the entire sampling period from regions with $\dot{Q}^{+}>1$ so that only reacting regions are considered. These PDFs are obtained using about 1200 bins for $\mathcal{B}$ and 400 bins for $Z$. This joint-PDF is shown for case AZ1 in Fig. 8 for various axial locations. It is observed that lean mixtures have mostly negative $\mathcal{B}$ while very rich mixtures $(0.02 \leq Z \leq 0.08$, or $2 \leq \phi \leq 8.5)$ have positive $\mathcal{B}$. Furthermore, the range of $Z$ decreases as one moves downstream due to turbulent mixing and also the range of $\mathcal{B}$ decreases (see Fig. 8c) because regions with $\dot{Q}^{+}>1$ becomes almost negligible as seen in Fig. 2a. Thus, the PDF value for large $\mathcal{B}$ also drops at downstream locations.

Figure 9 shows the $\mathrm{PDF}$ of $\mathcal{B}$ conditioned on the large heat release rate computed at various axial locations for all three cases. It is observed that close to the inlet both ignition and flame regions are equally important for the case AZ1 (Fig. 9a) since the PDF shows a broad peak covering both negative and small positive values of $\mathcal{B}$. As one moves downstream, the PDF peak shifts quite clearly towards $\mathcal{B}=0$ with substantial increase in the PDF values for $0 \leq \mathcal{B}<3$ indicating that propagating flame phenomena is likely to be the most probable mode. However, a negative tail is still observed in downstream locations which suggests that ignition-like reaction zones are also present. This confirms that ignition and flame propagation are closely linked in space as observed in Fig. 2. The PDF for the case AZ2 shows a clear shift of its peak towards negative $\mathcal{B}$ values which indicates an increase in reaction dominated regions. The case BZ1 shows a behaviour similar to the case AZ1 with a peak at slightly negative $\mathcal{B}$ at first and it slowly shifts towards 0 as 


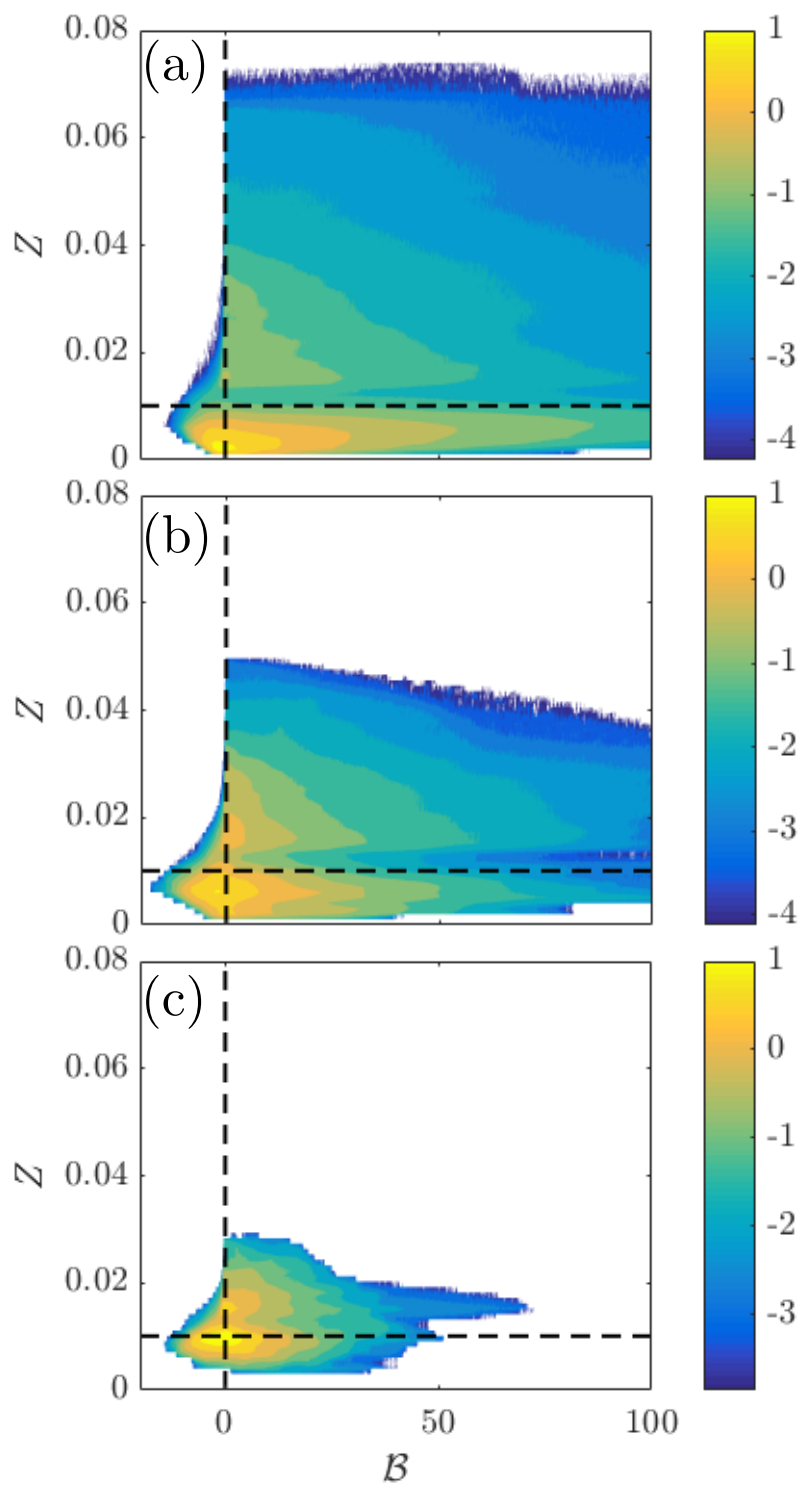

Fig. 8. Logarithm of the joint-PDF of $\mathcal{B}$ and $Z$ at (a) $x / L_{x}=0.0625$, (b) 0.4375 , (c) 0.9375 for the case AZ1. The horizontal line indicates $Z_{s t}$.

one moves downstream. The range of $\mathcal{B}$ variation in the negative region for the case BZ1 is smaller than for the other two cases (compare Fig. 9c with 9a 
and $9 \mathrm{~b}$ ). This is due to the lower level of oxygen (higher dilution) in the case BZ1 giving lower reaction rate as observed in [21,32]. Since the turbulent flow field is the same, the magnitude of convective-diffusive effects remains similar across all the cases and the magnitude of the reaction term in case BZ1 is lower yielding larger positive $\mathcal{B}$ on average.

The different behaviour observed for the case AZ2 shows the influence of the length-scale ratio, $\ell_{Z} / \ell_{c}$, on the ignition or flame attributes (PDF of $\mathcal{B}$ ). Indeed, the cases AZ1 and BZ1 with $\ell_{Z} / \ell_{c}>1$ have relatively smooth and low gradients of $Z$ since $\ell_{Z}$ is large. As a result, ignition occurs in regions with lean mixtures having low ignition delay and this will then develop into flames. The case AZ2 has smaller $\ell_{Z}$ (see Table 2) yielding increased mixture stratification. The presence of stronger gradients of $Z$ prevents such a development and favours sequential ignition [31]. Furthermore, a wider range of $Z$ in the case AZ2 (larger $\sigma_{Z}$ in Table 2) gives a larger range of ignition delay time and thus ignition occurs both in upstream and downstream locations. The mixtures outside the flammability limits mix with neighbouring mixtures before they can ignite [22] and thus these mixtures can ignite only in the downstream locations as illustrated earlier in Fig. 6. The PDF of $\mathcal{B}$ conditioned on $\dot{Q}^{+}>1$ depicted in Fig. 9b shows that the behaviour described above also holds in a statistical sense, which is confirmed further in Fig. 10b discussed below.

It is possible to further characterize the ignition regions by analysing the mixture fraction, $Z$, in these regions. Figure 10 shows the PDF $Z$ conditioned using $\mathcal{B}<-1$ at various axial locations. The condition $\mathcal{B}<-1$ instead of $\mathcal{B}<0$ is chosen to avoid plausible ambiguities that could arise 

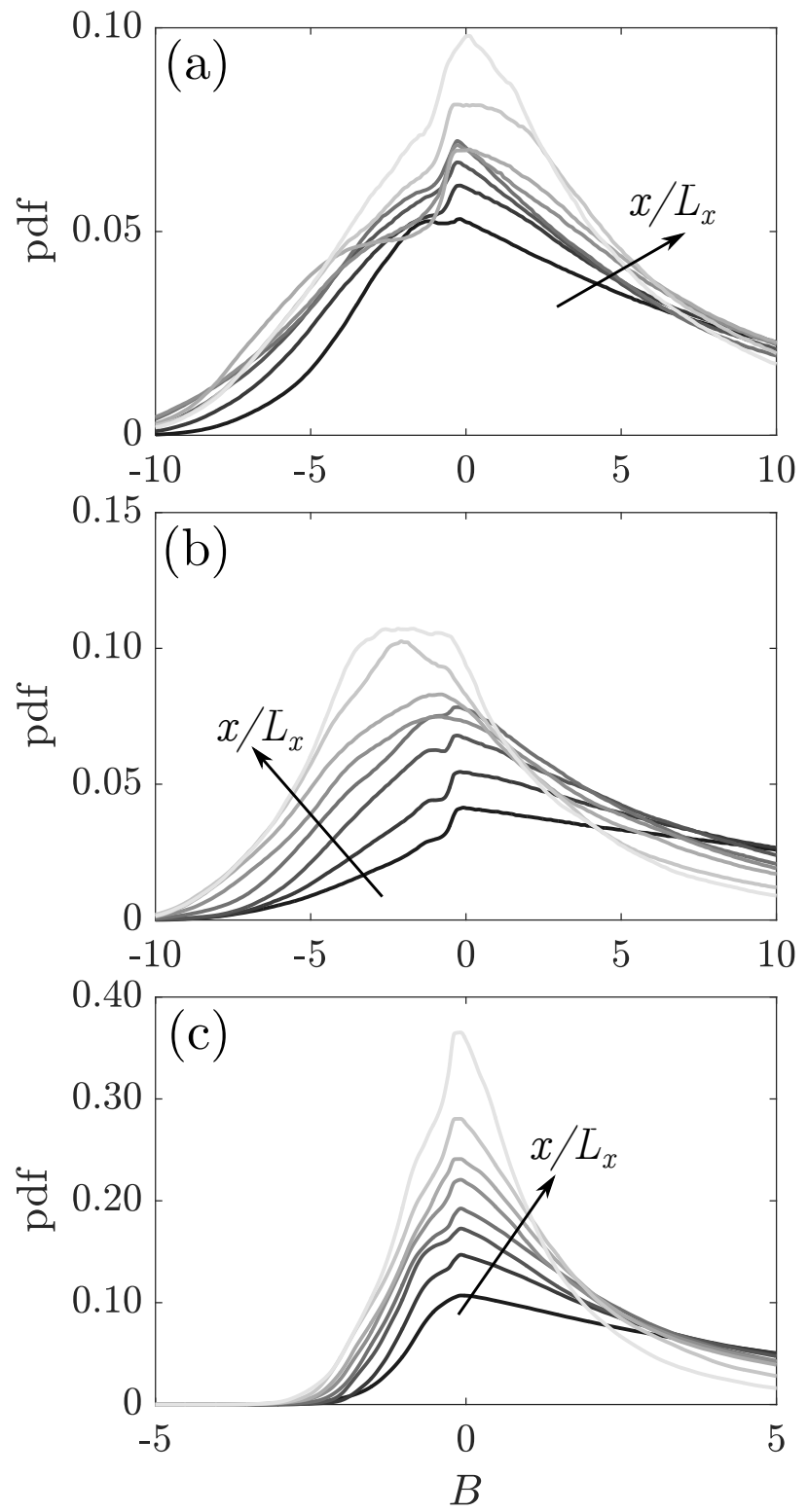

Fig. 9. PDF of $\left(\mathcal{B} \mid \dot{Q}^{+}>1.0\right)$ at $x / L_{x}=0.0625,0.1875,0.3125, \cdots, 0.9375$ (dark to light gray) for cases (a) AZ1, (b) AZ2 and (c) BZ1.

from flame-like regions with $-1 \leq \mathcal{B} \leq 0$. Thus, only strongly reaction dominated regions and their mixture conditions are identified using $\mathcal{B}<-1$ 

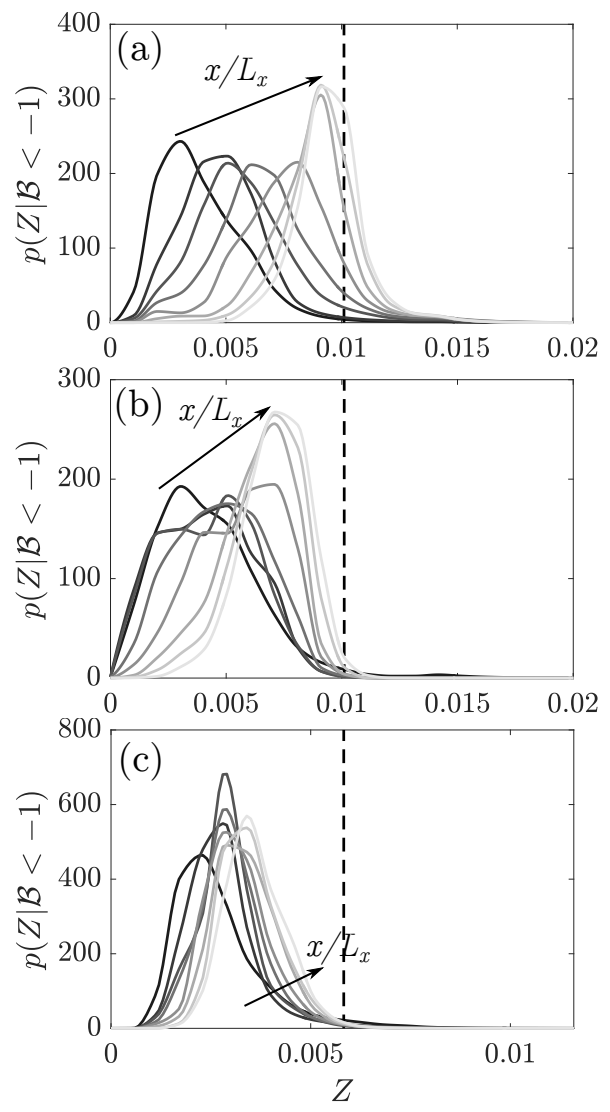

Fig. 10. PDF of $(Z \mid \mathcal{B}<-1.0)$ at $x / L_{x}=0.0625,0.1875,0.3125, \cdots, 0.9375$ (dark to light gray) for cases (a) AZ1, (b) AZ2 and (c) BZ1. The dashed lines indicate the stoichiometric mixture fraction.

condition. It is observed that the PDF peaks for a very lean mixture fraction, $Z \approx 0.002$ or $\phi \approx 0.2$ for locations close to the inlet (see Fig. 10b). This mixture fraction value is close to the most reactive mixture fraction. As one moves downstream, this peak shifts towards richer mixtures confirming the existence of sequential autoignition. The shift from leaner to richer mixture is explained by the increase in ignition delay times with $Z$ shown in Fig. 11. These ignition delay times were obtained using CANTERA and by 
considering a constant volume homogeneous reactor containing an appropriate mixture initially at $T_{r}=1500 \mathrm{~K}$. The construction of the PSR mixture follows the same procedure as the one used for the DNS initial field as described in section 2. Thus, the results in Fig. 11 are representative of the ignition delay time for the conditions employed for the DNS of non-premixed MILD combustion data investigated in this study.

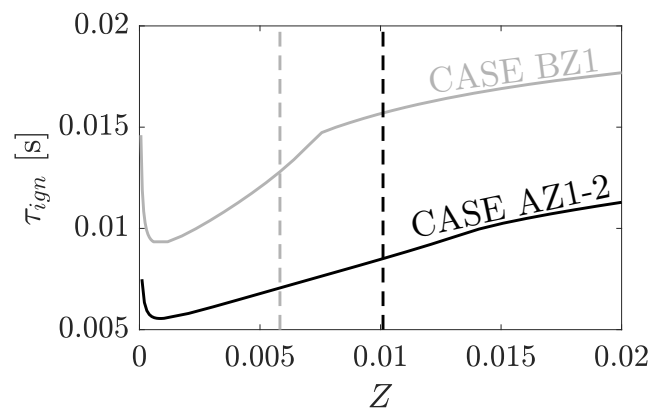

Fig. 11. Ignition delay time for the mixtures considered. The vertical dashed lines indicate the stoichiometric mixture fraction for the cases AZ1 and AZ2 (black) and BZ1 (gray).

A similar analysis is performed to characterise the mixtures in regions with steady flame-like structures in Fig. 12 for $-0.1<\mathcal{B} \leq 0.1$ and this range is denoted using $\mathcal{B} \approx 0$ in this figure. It is observed that the mixture in these regions in the case AZ1 evolve from lean to stoichiometric values indicating that as one moves downstream either flames start to appear in richer regions (from the apparition of richer ignition kernels as seen in Fig. 12) or that the existing leaner flames start to propagate into richer mixture. Furthermore, rich flames also exist in the downstream as indicated by the local peak of the $\mathrm{PDF}$ for richer mixture. These rich flames do not originate from the ignition of a rich kernel developing into a flame but from the propagation of a lean 
flame into those rich mixtures as the PDF of rich mixtures with $\mathcal{B}<-1$ is nearly zero as seen in Fig. 10a. This particular behaviour was also observed in the Lagrangian particles analysis studied in Fig. 5a. Similar observations can be made for the case BZ1, shown in Figs. 10c and 12c.

A different picture emerges for the case AZ2. It is observed in Fig. 12b that there already exist rich flames in the early stage of the domain. These rich flames originate from the propagation of lean flames towards richer mixtures and not from the ignition of rich kernels developing into flame-like structures since there is no ignition for $Z>Z_{s t}$ as shown in Fig. 10b depict-

ing $p(Z \mid B<-1) \approx 0$, for $Z>Z_{s t}$. Lean and rich mixtures are spatially closer in the case AZ2 because of its smaller $\ell_{Z}$, which allows for a lean flame to propagate into rich mixtures quickly compared to the cases AZ1 and BZ1 with $30 \%$ larger $\ell_{Z}$.

There are no rich flames appearing in downstream locations for the case AZ2. The spatial evolution of the mixture fraction PDF (not shown) shows that no rich mixtures are present at those downstream locations because the rich mixtures have already mixed with leaner mixtures. Since $\ell_{Z}$ is smaller in the case AZ2, the timescale required to mix and diffuse the rich regions is smaller.

\section{Summary and Conclusions}

DNS data of non-premixed MILD combustion are analysed to study the fundamental, specifically autoignition and flame propagation, aspects of this combustion. This analysis is conducted using nearly 1 million Lagrangian particles to track the evolution of fluid elements as they undergo ignition 

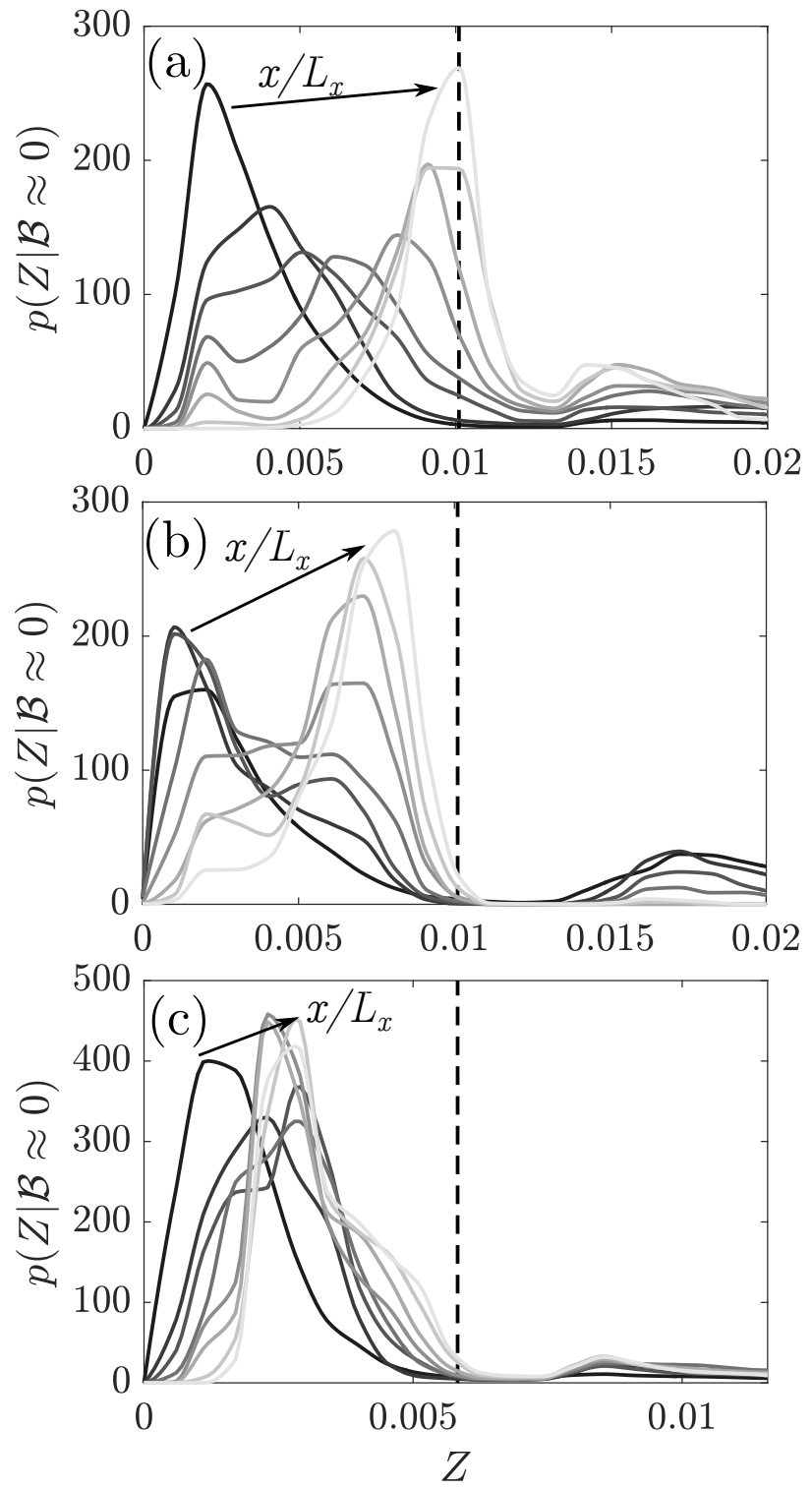

Fig. 12. PDF of $(Z \mid-0.1 \leq \mathcal{B} \leq 0.1)$ at $x / L_{x}=0.0625,0.1875,0.3125, \cdots, 0.9375$ (dark to light gray) for cases (a) AZ1, (b) AZ2 and (c) BZ1. The dashed line indicates the stoichiometric mixture fraction. 
or flame propagation under MILD conditions. These two phenomena are identified using the balance of various fluxes in the transport equation of $\mathrm{H}_{2} \mathrm{O}$ mass fraction. Similar results and conclusions are obtained using other species and thus the summary provided below is not dependent on the use of $\mathrm{H}_{2} \mathrm{O}$ mass fraction.

The picture that emerges from this study for the non-premixed MILD combustion is as follows. The lean mixtures ignite first in the upstream part of the computational domain and this is because of the low ignition delay times of these mixtures (see Fig. 11). The subsequent evolution of these kernels depends on the length scale ratio $\ell_{Z} / \ell_{c}$. When the mixture fraction length scale $\ell_{Z}$ is larger than $\ell_{c}$, the mixture is homogeneous promoting the development of flames from the kernels. These flames then propagate into rich and richer mixtures as they evolve in space and time. The stratified mixture ensuing when $\ell_{Z} \approx \ell_{c}$ promotes sequential ignition - ignition of leaner (most reactive) mixtures at the upstream locations which gradually propagates into rich mixture as one moves downstream. Also, the ignition of lean mixtures at an upstream axial location could produce rich flames at the same axial location depending on the dilution level. If the dilution is large (2\% compared to $5 \%$ oxygen mole fraction in the diluted oxidiser) then the probability to have rich flames are very small and MILD combustion occurs through ignition of lean mixtures and lean flames. Hence, non-premixed MILD combustion shows a variety of thermochemical phenomena which are also interacting with one another and thus the modelling of MILD combustion requires care. Assessing this will be a topic for future work. 


\section{Acknowledgements}

N.A.K.D. acknowledges the financial support of the Qualcomm European Research Studentship Fund in Technology. This work used the ARCHER UK National Supercomputing Service (http://www.archer.ac.uk) using computing time provided by EPSRC under the project number e419 and the UKCTRF (e305).

\section{References}

[1] J. A. Wünning, J. G. Wünning, Flameless oxidation to reduce thermal no-formation, Prog. Energy Combust. Sci. 23 (1) (1997) 81-94. doi:10.1016/S0360-1285(97)00006-3.

[2] A. Cavaliere, M. de Joannon, Mild Combustion, Prog. Energy Combust. Sci. 30 (4) (2004) 329-366. doi:10.1016/j.pecs.2004.02.003.

[3] T. Plessing, N. Peters, J. G. Wünning, Laseroptical investigation of highly preheated combustion with strong exhaust gas recirculation, 27th Symp. Combust. 27 (1998) 3197-3204. doi:10.1016/S00820784(98)80183-5.

[4] M. de Joannon, A. Saponaro, A. Cavaliere, Zero-dimensional analysis of diluted oxidation of methane in rich conditions, Proc. Combust. Inst. 28 (2) (2000) 1639-1646. doi:10.1016/S0082-0784(00)80562-7.

[5] I. B. Ozdemir, N. Peters, Characteristics of the reaction zone in a combustor operating at mild combustion, Exp. Fluids 30 (6) (2001) 683-695. doi:10.1007/s003480000248. 
[6] C. Duwig, B. Li, Z. S. Li, M. Aldén, High resolution imaging of flameless and distributed turbulent combustion, Combust. Flame 159 (1) (2012) 306-316. doi:10.1016/j.combustflame.2011.06.018.

[7] P. R. Medwell, P. A. M. Kalt, B. B. Dally, Simultaneous imaging of OH, formaldehyde, and temperature of turbulent nonpremixed jet flames in a heated and diluted coflow, Combust. Flame 148 (1-2) (2007) 48-61. doi:10.1016/j.combustflame.2006.10.002.

[8] J. A. M. Sidey, E. Mastorakos, R. L. Gordon, Simulations of Autoignition and Laminar Premixed Flames in Methane/Air Mixtures Diluted with Hot Products, Combust. Sci. Technol. 186 (4-5) (2014) 453-465. doi:10.1080/00102202.2014.883217.

[9] M. Katsuki, T. Hasegawa, The science and technology of combustion in highly preheated air, 27th Symp. Combust. 27 (1998) 3135-3146. doi:10.1016/S0082-0784(98)80176-8.

[10] P. R. Medwell, P. A. M. Kalt, B. B. Dally, Imaging of diluted turbulent ethylene flames stabilized on a Jet in Hot Coflow (JHC) burner, Combust. Flame 152 (1-2) (2008) 100-113. doi:10.1016/j.combustflame.2007.09.003.

[11] E. Oldenhof, M. J. Tummers, E. H. van Veen, D. J. E. M. Roekaerts, Ignition kernel formation and lift-off behaviour of jetin-hot-coflow flames, Combust. Flame 157 (6) (2010) 1167-1178. doi:10.1016/j.combustflame.2010.01.002. 
[12] J. A. M. Sidey, E. Mastorakos, Visualization of MILD combustion from jets in cross-flow, Proc. Combust. Inst. 35 (3) (2015) 3537-3545. doi:10.1016/j.proci.2014.07.028.

[13] E. Abtahizadeh, A. V. Sepman, F. Hernández-Pérez, J. A. van Oijen, A. V. Mokhov, L. P. H. de Goey, H. B. Levinsky, Numerical and experimental investigations on the influence of preheating and dilution on transition of laminar coflow diffusion flames to Mild combustion regime, Combust. Flame 160 (11) (2013) 2359-2374. doi:10.1016/j.combustflame.2013.05.020.

[14] E. Mastorakos, Ignition of turbulent non-premixed flames, Prog. Energy Combust. Sci. 35 (1) (2009) 57-97. doi:10.1016/j.pecs.2008.07.002.

[15] J. A. van Oijen, Direct numerical simulation of autoigniting mixing layers in MILD combustion, Proc. Combust. Inst. 34 (1) (2013) 1163-1171. doi:10.1016/j.proci.2012.05.070.

[16] M. U. Göktolga, J. A. van Oijen, L. P. H. de Goey, 3D DNS of MILD combustion: A detailed analysis of heat loss effects, preferential diffusion, and flame formation mechanisms, Fuel 159 (2015) 784-795. doi:10.1016/j.fuel.2015.07.049.

[17] Y. Minamoto, T. D. Dunstan, N. Swaminathan, R. S. Cant, DNS of EGR-type turbulent flame in MILD condition, Proc. Combust. Inst. 34 (2) (2013) 3231-3238. doi:10.1016/j.proci.2012.06.041.

[18] Y. Minamoto, N. Swaminathan, R. S. Cant, T. Leung, Reaction Zones 
and Their Structure in MILD Combustion, Combust. Sci. Technol. 186 (8) (2014) 1075-1096. doi:10.1080/00102202.2014.902814.

[19] Y. Minamoto, N. Swaminathan, R. S. Cant, T. Leung, Morphological and statistical features of reaction zones in MILD and premixed combustion, Combust. Flame 161 (11) (2014) 2801-2814. doi:10.1016/j.combustflame.2014.04.018.

[20] Y. Minamoto, N. Swaminathan, Scalar gradient behaviour in MILD combustion, Combust. Flame 161 (4) (2014) 1063-1075. doi:10.1016/j.combustflame.2013.10.005.

[21] M. de Joannon, P. Sabia, G. Cozzolino, G. Sorrentino, a. Cavaliere, Pyrolitic and Oxidative Structures in Hot Oxidant Diluted Oxidant (HODO) MILD Combustion, Combust. Sci. Technol. 184 (7-8) (2012) 1207-1218. doi:10.1080/00102202.2012.664012.

[22] N. A. K. Doan, N. Swaminathan, Y. Minamoto, DNS of MILD combustion with mixture fraction variations, Combust. Flame 189 (2018) 173-189. doi:https://doi.org/10.1016/j.combustflame.2017.10.030.

[23] R. W. Bilger, S. H. Starner, R. J. Kee, On Reduced Mechanisms for Methane-Air Combustion in Nonpremixed Flames, Combust. Flame 80 (1990) 135-149.

[24] V. Eswaran, S. B. Pope, An examination of forcing in direct numerical simulations of turbulence, Comput. Fluids 16 (3) (1988) 257-278.

[25] G. R. Ruetsch, M. R. Maxey, Small-scale features of vorticity and passive 
scalar fields in homogeneous isotropic turbulence, Phys. Fluids A 3 (6) (1991) 1587-1597. doi:10.1063/1.857938.

[26] P. K. Yeung, S. B. Pope, Differential diffusion of passive scalars in isotropic turbulence, Phys. Fluids A 5 (10) (1993) 2467-2478. doi:10.1063/1.858760.

[27] M. D. Smooke, V. Giovangigli, Formulation of the premixed and nonpremixed test problems, in: M. D. Smooke (Ed.), Reduc. Kinet. Mech. Asymptot. Approx. Methane-Air Flames, Vol. 384 of Lecture Notes in Physics, Springer Berlin Heidelberg, Berlin/Heidelberg, 1991, pp. 1-28. doi:10.1007/BFb0035362.

[28] T. Kathrotia, U. Riedel, A. Seipel, K. Moshammer, A. Brockhinke, Experimental and numerical study of chemiluminescent species in lowpressure flames, Appl. Phys. B Lasers Opt. 107 (3) (2012) 571-584. doi:10.1007/s00340-012-5002-0.

[29] T. Poinsot, Boundary conditions for direct simulations of compressible viscous flows, J. Comput. Phys. 101 (1992) 104-129. doi:10.1016/00219991(92)90227-P.

[30] N. A. K. Doan, N. Swaminathan, Role of radicals on MILD combustion inception, Proc. Combust. Inst. 37 (2019) (available online, https://doi.org/10.1016/j.proci.2018.07.038).

[31] M. B. Luong, G. H. Yu, S. H. Chung, C. S. Yoo, Ignition of a lean PRF/air mixture under RCCI/SCCI conditions: 
Chemical aspects, Proc. Combust. Inst. 36 (3) (2017) 3587-3596. doi:10.1016/j.proci.2016.08.038.

[32] M. de Joannon, A. Cavaliere, T. Faravelli, E. Ranzi, P. Sabia, A. Tregrossi, Analysis of process parameters for steady operations in methane mild combustion technology, Proc. Combust. Inst. 30 (2) (2005) 26052612. doi:10.1016/j.proci.2004.08.190. 